\title{
Efecto inmediato de dos ejercicios con tracto vocal semiocluido en el contacto glótico de usuarios ocupacionales de la voz
} Immediate effect of two semi-occluded vocal tract exercises in
glottal contact of occupational voice users

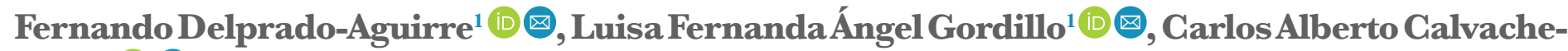
Mora $^{2}$ (i) 중

${ }^{1}$ Facultad de Medicina; Universidad Nacional de Colombia; Bogota; Colombia.

${ }^{2}$ Vocology Center; Bogotá; Colombia.

\section{D) Check for updates}

\section{Correspondencia}

Fernando Delprado Aguirre.

Email: afdelpradoa@unal.edu.co

\section{Citar así}

Delprado Aguirre, Fernando; Ángel Gordillo, Luisa Fernanda; Calvache-Mora, Carlos Alberto. (2022). Efecto inmediato de dos ejercicios con tracto vocal semiocluido en el contacto glótico de usuarios ocupacionales de la voz. Revista de Investigación e Innovación en Giencias de la Salud. 4(1), 26-42. https://doi. org/10.46634/riics. 76

\section{Recibido: 21/07/2021}

Revisado: 07/09/2021

Aceptado: 23/11/2021

\section{Editor}

Jorge Mauricio Cuartas Arias, Ph.D. iD

\section{Coeditor}

Fraidy-Alonso Alzate-Pamplona, MSc.

Copyright $($ 2022. Fundación Universitaria María Cano. The Revista de Investigación e

Innovación en Ciencias de la Salud provides open access to all its content under the terms of the Creative Commons Attribution-

NonCommercial-NoDerivatives 4.0

International (CG BY-NG-ND 4.0).

\section{Declaración de intereses}

Los autores han declarado que no hay conflicto de intereses.

\section{Resumen}

Introducción. Los docentes son usuarios ocupacionales de la voz con alto riesgo de desarrollar patologías vocales a causa de su labor. Para enfrentar esta problemática, suelen usarse ejercicios con tracto vocal semiocluido (TVSO), estrategia de educación/rehabilitación empleada para generar un cambio en el patrón de vibración de los pliegues vocales, minimizando el riesgo de lesión vocal al reducir el estrés de colisión al que se someten los tejidos. Diversos reportes han indicado que este tratamiento tiene efectos en el cociente de cierre (CQ), medida indirecta del estrés de colisión.

Objetivo. Examinar el efecto fisiológico de dos ejercicios con TVSO en la actividad laríngea de profesores con esfuerzo vocal constante pero sin patología vocal.

Metodología. Se registraron muestras de 43 profesores antes, durante y después de la realización de dos ejercicios con TVSO (vibración lingual y fonación en tubos). Las muestras de electroglotografía se analizaron para obtener el CQ.

Resultados y conclusión. Se observó una diferencia significativa al comparar los valores del CQ antes y durante la realización de ambos ejercicios. No se encontraron cambios en este parámetro después de ejecutar la actividad. Estos hallazgos concuerdan con reportes previos en los cuales el CQ tiende a aumentar durante la fonación en tubos; contrariamente, la realización de la vibración lingual tiende a decrecer el valor del CQ. Es posible que este comportamiento se deba a los mecanismos biomecánicos particulares de cada ejercicio.

\section{Palabras clave}

Tracto vocal semiocluido; fonación en tubos; vibración lingual; electroglotografía; cociente de cierre; laringe; pliegues vocales; fisiología de la voz; entrenamiento de la voz; vocología. 
Disponibilidad de datos

Todos los datos relevantes se encuentran en el artículo. Para mayor información, comunicarse con el autor de correspondencia.

\section{Financiamiento}

Ninguno. Esta investigación no recibió ninguna subvención específica de agencias de financiamiento en los sectores público, comercial o sin fines de lucro.

\section{Descargo de responsabilidad} El contenido de este artículo es responsabilidad exclusiva de los autores y no representa una opinión oficial de sus instituciones ni de la Revista de Investigación e Innovación en Ciencias de la Salud.

\section{Agradecimientos}

A Martha Peña y Alejandro Múnera por sus valiosas contribuciones en el diseño metodológico de este estudio

\section{Contribución de los autores} Fernando Delprado Aguirre: conceptualización, curación de datos, análisis formal, investigación, metodología, administración de proyecto, recursos, software, validación, visualización, escritura: borrador original, escritura: revisión y edición. Luisa Fernanda Ángel Gordillo: conceptualización, curación de datos, metodología, administración de proyecto, recursos, supervisión, validación, visualización, escritura: borrador original. Carlos Alberto Calvache-Mora: metodología, administración de proyecto, recursos, visualización, escritura: borrador original.

\section{Abstract}

Introduction. Teachers are occupational voice users with high risk of developing vocal pathologies due to their work. To face this situation, it is common the use of semi-occluded vocal tract (SOVT) exercises, a strategy of voice education/rehabilitation implemented to induce a change in the vibration pattern of the vocal folds, mitigating, therefore, the risk of vocal lesion by reducing the collision stress applied to tissues. A variety of reports have indicated that this treatment has effects in the closed quotient $(\mathrm{CQ})$, an indirect measure of collision stress.

Aim. The purpose of this study was to examine the physiological effect of two different SOVT exercises in larynx activity of teachers with constant vocal effort but without vocal pathology.

Methods. 43 samples of teachers were recorded before, during and after executing two SOVT exercises (tongue trill and tube phonation). Electroglottographic samples were analyzed in order to obtain CQ.

Results and conclusion. Both exercises had a significant difference of CQ scores when before and during conditions were compared. Any difference was found on this parameter after executing the activity. These findings agree with previous reports where CQ tends to increase during phonation through resonance tubes; on the contrary, execution of tongue trill tends to decrease CQvalues. This behavior might be because of the particular biomechanical mechanisms of each exercise.

\section{Keywords}

Semi-occluded vocal tract; resonance tubes; tongue trill; electroglottography; contact quotient; larynx; vocal cords; voice physiology; voice training; vocology.

\section{Introducción}

Los individuos que dependen de sus voces para funcionar exitosamente en sus ocupaciones son considerados usuarios ocupacionales de la voz [1]. Según las estimaciones de Vilkman [2], este grupo corresponde a un tercio de la población mundial, al cual se le han acentuado los problemas crónicos del mecanismo vocal a través de los años, especialmente en los docentes, quienes tienen más riesgo de desarrollar desórdenes de voz en comparación con otros profesionales de la voz [3,4]. De acuerdo con estimaciones internacionales, la prevalencia de desórdenes vocales en los docentes puede alcanzar cifras del 80\% [5]. Para el contexto colombiano, el efecto del desorden vocal es tal, que - según lo reportado por Cantor-Cutiva et al. [6] - los costos por concepto de atención médica y productividad relacionados con la voz de un docente equivalen aproximadamente al 37\% del salario mínimo.

Tradicionalmente, los programas de higiene vocal, la amplificación vocal y la terapia confidencial han liderado como estrategias terapéuticas para esta población [7]. No obstante, el entrenamiento orientado a la economía vocal tiene su principal aplicación en sujetos que sufren los efectos de usar la voz por tiempos prolongados [8]. Este tipo de entrenamiento está basado en la premisa de que la lesión vocal, o el riesgo de desarrollarla, puede minimizarse si las dosis de vibración y el estrés de colisión se reducen, provocando menor daño mecánico a los tejidos. 
Una de las estrategias más populares de tratamiento/educación basadas en la economía vocal son los ejercicios con TVSO. Estos ejercicios comprenden un grupo de maniobras que involucran la constricción parcial y/o el alargamiento artificial del tracto vocal para generar un cambio en el patrón de vibración de los pliegues vocales [9]. Entre los objetivos principales para la ejecución de ejercicios con TVSO se encuentran: 1) liberar el esfuerzo mecánico al que se someten los pliegues vocales durante la fonación y 2) aumentar el poder acústico del producto vocal, basándose en la interacción fuente-filtro y no en el estrés de colisión de los pliegues vocales [10]. Guzmán et al. [11] y Laukkanen et al. [12] han explicado este cambio como consecuencia del incremento en la presión intraoral durante la adopción de las distintas posturas, generando un efecto de empuje en dirección posterior hacia la laringe y las paredes laterales de la faringe, lo que separa ligeramente los pliegues vocales durante la fonación [13].

Existen diversas clasificaciones de los ejercicios con TVSO [14-16]. Una de ellas considera el número de fuentes de vibración, de forma tal que en los ejercicios de una fuente de vibración solamente oscilan los pliegues vocales, esto es, nasales $[17,18]$, zumbidos $[19,20]$, fonación en tubos [8] y manipulaciones [21]. Además de los pliegues vocales, en los ejercicios de dos fuentes de vibración se observa la oscilación de otros órganos. Por ejemplo, vibraciones $[22,23]$ o dispositivos (a saber, terapia de resistencia en el agua [24] o dispositivos de presión positiva [25]). En el contexto colombiano, dos de los ejercicios más usados, por la disponibilidad comercial de los instrumentos y por la cercanía lingüística con los fonemas del español, son la fonación en tubos y la vibración labial.

$\mathrm{Al}$ estudiar los efectos del ejercicio de fonación en tubos en el proceso fonatorio (mediante electroglotografia), se encontraron resultados que discrepan entre sí. Inicialmente, Laukkanen [26] encontró que la realización de estos ejercicios modificaba los registros electroglotográficos, al aumentar el valor del cociente de velocidad y disminuir los valores del cuasi-cociente de apertura (QOQ), lo cual indicaría un mayor contacto entre los pliegues vocales. De acuerdo con la autora, esta mejoría de la función vocal se debe a una retroalimentación mecano-acústica del tracto vocal sobre los pliegues vocales. En años más recientes, Gaskill et al. [27] no evidenciaron ningún patrón de cambio, mientras que en otro estudio del mismo investigador [28] se observó una tendencia al aumento del CQ durante la ejecución del ejercicio. De forma opuesta, Guzmán et al. [11] encontraron una disminución clínica más no significativa de la misma variable. Los autores indican que los resultados particulares en cada uno de los estudios pueden deberse a factores como el nivel de entrenamiento o la metodología de instrucción [27], a la longitud de los tubos o a las tareas fonatorias desarrolladas con el ejercicio [28] o, quizás, al tiempo de realización del ejercicio [11].

Igualmente, los efectos de la vibración labial y lingual han sido estudiados por medio de mediciones electroglotográficas. Gaskill et al. [29] y Hamdan et al. [30] observaron una reducción del CQ durante la realización de los ejercicios, siendo más evidente para los sujetos sin entrenamiento [29,30]. En contraposición, Cordeiro et al. [23] observaron que el CQ aumentaba durante la realización de ambos ejercicios, especialmente a altas intensidades. Frente a esto, los autores afirmaron que era esperable un aumento en el contacto glótico, puesto que durante la realización del ejercicio aumentaba la presión subglótica y, en consecuencia, ocurría también un aumento de la aducción glótica.

Ahora bien, al analizar conjuntamente diversos ejercicios, Guzmán et al. [31] y Andrade et al. [15] demostraron que las mediciones del CQ durante la fonación variaban dependiendo del ejercicio. Los investigadores observaron un aumento de esta medida en ejercicios como la 
terapia de resistencia en el agua, los zumbidos y la mano sobre la boca. Contrariamente, el CQ disminuyó durante la ejecución de las vibraciones [31]. Por su parte, Andrade et al. [15] demostraron que en los ejercicios de una sola fuente, el parámetro es relativamente estable, mientras que para los ejercicios de dos fuentes, el parámetro fluctúa durante su ejecución. La conclusión general de estos autores es que una baja variación del CQ favorece una fonación fácil, en cambio, una alta variación del CQ tiene mayor "efecto masaje", pero la voz requiere más esfuerzo.

Partiendo de estos hallazgos, resulta conveniente investigar los efectos que estos ejercicios producen en la fisiología fonatoria de los profesores. Según Cantor-Cutiva et al. [3] esta población se encuentra en mayor riesgo de desarrollar desórdenes de voz, dado que no suelen contar con entrenamiento previo para usar su voz adecuadamente. En ese sentido, un tratamiento basado en la economía vocal puede ser benéfico para esta población. Hasta la fecha, solo algunos estudios como el de Mailänder et al. [32] han evaluado los efectos fisiológicos del TVSO específicamente en docentes, demostrando resultados positivos. A nivel local, los estudios de Calvache [33,34] examinaron los efectos de los ejercicios con TVSO, pero evaluando a sujetos entrenados o con patologías vocales. Por lo tanto, estas estrategias deben explorarse en el contexto colombiano. Así pues, el propósito de este estudio fue determinar el efecto fisiológico de dos ejercicios con TVSO en la actividad laríngea de profesores con esfuerzo vocal constante pero sin patología vocal.

\section{Metodología}

\section{Participantes}

Este estudio prospectivo analítico con diseño pretest-postest fue aprobado por el Comité de Ética de la Facultad de Medicina de la Universidad Nacional de Colombia bajo el acta 012 210-18. Participaron un total de 43 voluntarios del género masculino; no se tuvo en cuenta la población del género femenino debido a los cambios hormonales que pueden impactar la función vocal [35]. El tamaño de muestra para este estudio se determinó con un coeficiente de correlación temporal del 0.6, a un nivel de confianza del $95 \%$ y con una potencia del $80 \%$. El coeficiente de correlación temporal se obtuvo a partir de los datos de Guzmán et al. [31]. Los participantes fueron profesores sanos, a pesar del esfuerzo vocal constante y sin entrenamiento vocal previo. Fueron reclutados por convocatoria abierta, mediante volantes de difusión, y se encontraban vinculados a diferentes instituciones de educación superior en donde ofrecían cátedras magistrales. Estos podían o no contar con pautas de higiene vocal. La totalidad de los participantes cumplieron con los siguientes criterios de inclusión: 1) rango de edad de 25 a 45 años; 2) no haber contado con terapia o entrenamiento vocal; 3) sin historia pasada o actual de desórdenes de voz, lo cual se corroboró con una puntuación menor a 18 en el Índice de Discapacidad de la Voz [36] y puntuaciones de 0 en la escala perceptual auditiva GRBAS; 4) tiempo de uso vocal mayor a 20 horas a la semana. Los criterios de exclusión fueron: 1) diagnóstico de lesiones en el pliegue vocal, 2) ser fumadores, 3) presentar cuadros gripales en el momento de la toma de la muestra, 4) imposibilidad de realizar los ejercicios de forma adecuada y 4) reporte de alergias al material de los electrodos de electroglotografía (EGG). Todos los participantes eran hablantes nativos del español. 


\section{Ejercicios y tareas de voz}

Antes del registro de datos, los participantes diligenciaron el consentimiento informado y el Índice de Discapacidad de la Voz $[37,38]$. El registro de datos se inició con tomas acústicas y de electroglotografía en tres momentos (antes, durante y después de cada ejercicio). Para ello, los participantes emitieron la vocal /a:/ por 5 segundos en tono e intensidad habituales antes y después del ejercicio. Así mismo, mientras los participantes ejecutaban la semioclusión, se realizaron registros electroglotográficos. La emisión del tono fue monitoreada perceptualmente por el evaluador. En los momentos previo y posterior al ejercicio se capturaron tres repeticiones de la vocal con el ánimo de obtener un promedio de esas producciones. De igual forma, se les solicitó mantener una emisión relajada durante todo el protocolo. Los registros durante el ejercicio se realizaron en los minutos 1,3 y 5 .

Los ejercicios evaluados fueron: 1) fonación en tubos y 2) vibración lingual, ejecutando la tarea fonatoria de tono sostenido. Estos fueron seleccionados teniendo en cuenta su alto uso en el país y su especial cercanía con el idioma español. Antes de cada ejercicio se ofreció un modelamiento por parte de uno de los investigadores. Para la fonación en tubos el sujeto debía sostener un tubo plástico/mezclador $(10 \mathrm{~cm}$ de longitud y $4 \mathrm{~mm}$ de diámetro $)$ firmemente entre los labios. Se instruyó al voluntario para mantener un selle completo de los labios alrededor del tubo mientras emitía un sonido similar a la vocal /u/. Para la vibración lingual se instruyó a los pacientes en la prolongación de un sonido similar a la /r/ del español. Ambos ejercicios se realizaron durante 5 minutos continuos, tomando las pausas necesarias para respirar. Las tareas fonatorias, monitoreadas por el evaluador, se ejecutaron en tono e intensidad habituales. Durante los ejercicios se indicó a los voluntarios que se concentraran en las sensaciones de vibración en la región facial, así como en la facilidad para producir el sonido. Los investigadores ofrecieron retroalimentación, siguiendo los principios de aprendizaje sensoriomotor. Dado que este fue un diseño crossover, 21 participantes iniciaron el experimento con la fonación en tubos y los restantes 22 iniciaron el experimento con la vibración labial. Entre cada uno de los ejercicios los sujetos mantuvieron 15 minutos de reposo vocal.

\section{Equipos}

Todo el protocolo de evaluación se realizó en las oficinas de los docentes, considerando que estos eran sus ambientes naturales. Los participantes se ubicaron en una silla cómoda y permanecieron en posición sedente durante toda la examinación. Inmediatamente después, se limpió la piel del cuello de los participantes, así como la superficie de los electrodos con un paño húmedo. Estos se ubicaron sobre cada una de las alas del cartílago tiroides, y se ajustaron firmemente mediante el uso de una banda de velcro para evitar su desplazamiento durante los registros.

La evaluación electroglotográfica y acústica se realizó con un electroglotógrafo de 1 canal (Electroglottograph modelo 7050A, EGGs for singers, Holanda, 2018). Las muestras se grabaron digitalmente en archivos.VIS a una taza de muestreo de $44 \mathrm{kHz}$ y 16 bits. De los registros electroglotográficos obtenidos antes, durante y después de la realización de los ejercicios, se extrajo el CQ [39]. El CQ se calculó automáticamente mediante el software VoceVistaPro 3.2 (VoceVista, 2018), seleccionando la zona media más estable de cada muestra. Para este cálculo se seleccionó un nivel de criterio estándar de 35\% de la amplitud pico a pico en la curva electroglotográfica según la propuesta de Howard [39], Miller [40], y Rothenberg y Mahshie [41] para el análisis de voces normales. 


\section{Análisis de datos}

Para calcular las diferencias en el CQ antes, durante y después de la realización de los ejercicios, se utilizó un diseño crossover pretest-postest. Teniendo en cuenta que las pruebas de Shapiro-Wilk indicaron que los datos presentaban una distribución normal aproximada, la comparación univariada se realizó mediante la prueba t-pareada. En total se realizaron cuatro comparaciones en los cálculos del CQ: dos pruebas entre los resultados antes y después de la realización de cada ejercicio y dos pruebas más para comparar los resultados antes y durante la realización de los ejercicios. Todas las pruebas se realizaron con un nivel de confianza del 95\%; los valores p reportados fueron a dos colas.

Posteriormente, con los datos obtenidos del experimento, se ajustó un modelo de análisis de varianza (ANOVA) para un diseño crossover, en el que la variable respuesta fue el valor del CQy las variables explicativas eran el tratamiento (fonación en tubos y vibración labial), el periodo (orden de realización de los ejercicios) y la interacción entre estas dos. Todos los análisis descritos se ejecutaron en el software estadístico R.

\section{Resultados}

\section{Comportamiento del CQ antes, durante y después de los ejercicios} El promedio del CQ en los tres momentos de medición para cada uno de los ejercicios ejecutados se presenta en la Tabla 1. Adicionalmente, las Figuras 1 y 2 muestran la distribución del CQ en los tres momentos de medición y según cada ejercicio estudiado.

\begin{tabular}{|c|c|c|c|}
\hline & Pre $\pm \mathrm{DE}$ | CV & Durante $\pm \mathrm{DE}$ | CV & Pos \pm DE I CV \\
\hline Fonación en tubos & $0.492 \pm 0.048 \mid 9.8 \%$ & $0.518 \pm 0.047 \mid 9 \%$ & $0.489 \pm 0.045 \mid 9.2 \%$ \\
\hline Vibración lingual & $0.48 \pm 0.052 \mid 10.8 \%$ & $0.441 \pm 0.047 \mid 10.6 \%$ & $0.483 \pm 0.05 \mid 10.4 \%$ \\
\hline
\end{tabular}

Nota: Los datos se presentan como media \pm desviación estándar | coeficiente de variación.

Se obtuvo una diferencia estadísticamente significativa al analizar los valores del CQ registrados antes y durante la fonación en tubos $(p<0.001)$. No obstante, al considerar los resultados antes y después de la realización del mismo ejercicio, no se obtuvo suficiente evidencia estadística para rechazar la H0 $(p=0.666)$. Ahora bien, al examinar los valores del CQ antes, durante y después de la ejecución de la vibración lingual, se observó un comportamiento similar al obtenido con el primer ejercicio. Cuando se compara el CQ antes y durante, existe suficiente evidencia estadística para rechazar la $\mathrm{H} 0$, de modo que se confirma la diferencia estadísticamente significativa $(p<0.001)$. Por otro lado, al tener en cuenta los valores del CQ antes y después, no se evidenciaron diferencias significativas $(p=0.715)$. Además, es necesario mencionar que la dirección de cambio del CQ es diferente durante la realización de ambos ejercicios: mientras que el CQ aumentó durante la fonación en tubos, el mismo parámetro disminuyó cuando los sujetos realizaron la vibración lingual. 
Efecto de dos ejercicios con tracto vocal semiocluido

Delprado Aguirre et al.

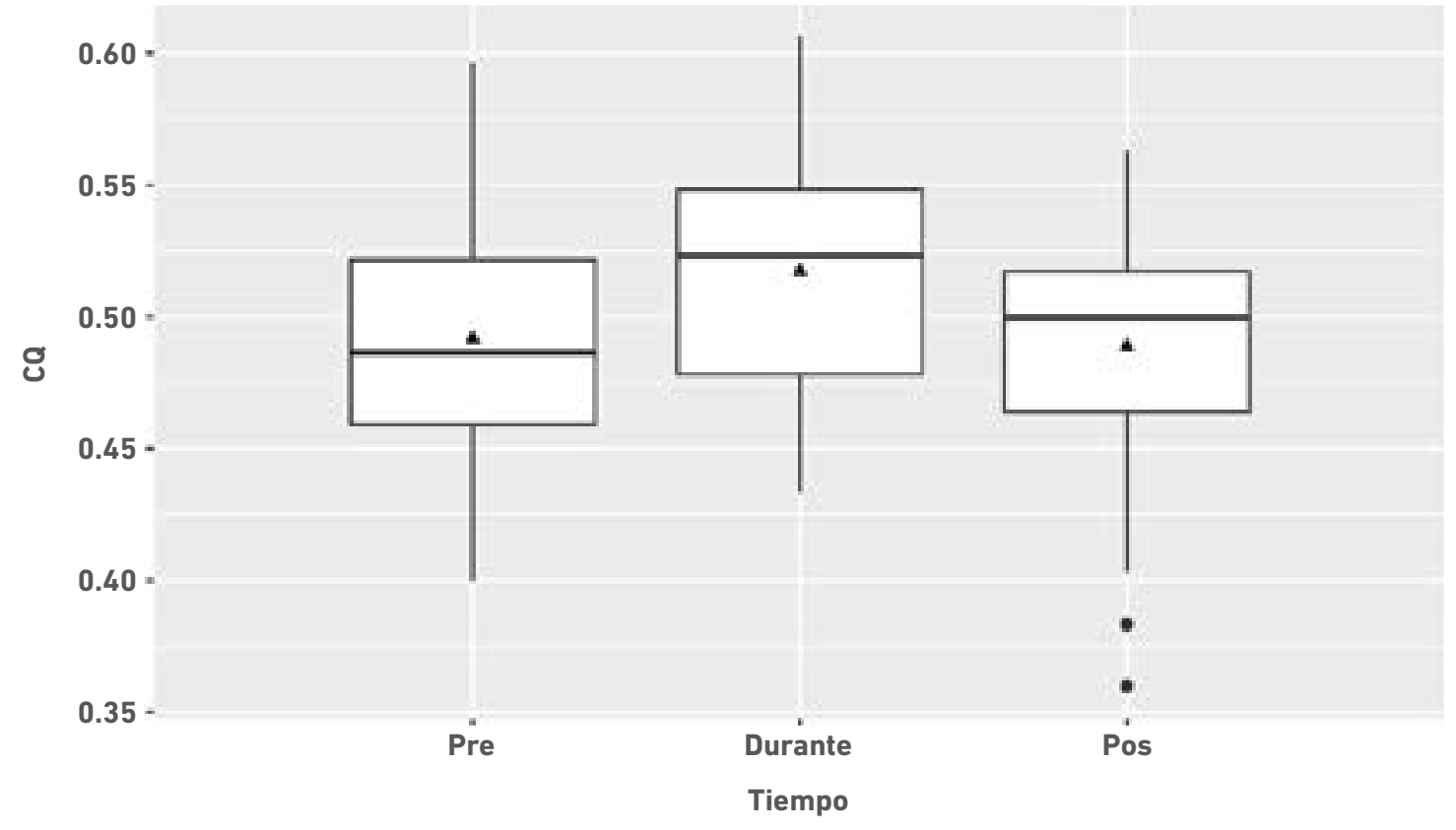

Figura 1. Comportamiento del CQ para el ejercicio de fonación en tubos

Nota: Los triángulos representan el promedio de todos los participantes en cada uno de los momentos de medición.

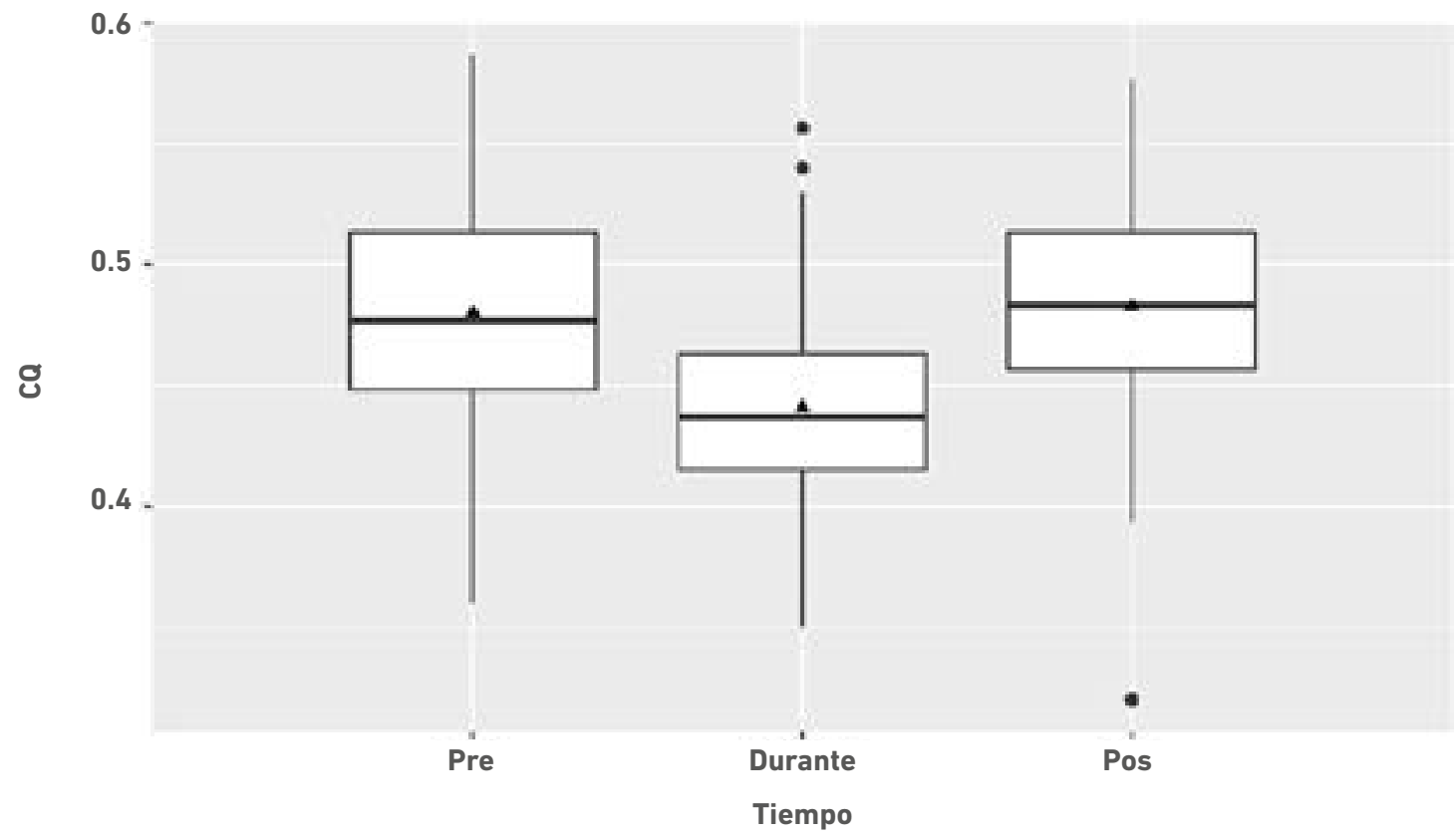

Figura 2. Comportamiento del $C Q$ para el ejercicio de vibración lingual

Nota: Los triángulos representan el promedio de todos los participantes en cada uno de los momentos de medición. 


\section{Efecto del periodo y el tratamiento sobre el CQ}

Luego de ajustar el modelo de análisis de varianza (ANOVA), se encontró que no existe efecto del tratamiento sobre la variable respuesta $(p=0.562)$, no existen efectos residuales de un tratamiento sobre el otro $(p=0.488)$ y el periodo de ejecución de los ejercicios tiene un efecto en los valores del CQ $(p<0.001)$.

\section{Modificaciones en el perfil de onda}

$\mathrm{Al}$ analizar las ondas electroglotográficas se apreciaron modificaciones en los perfiles de onda antes y durante la realización de los ejercicios. Para comenzar, durante la realización de la fonación en tubos, la consecución del contacto glótico máximo se logró más rápidamente, el perfil de onda se hizo más robusto durante la fase de cierre, lo cual provocó el aumento del CQ, y, adicionalmente, aunque la fase abierta tendió a ser más corta, exhibió una pendiente menos pronunciada en la zona de transición de la fase abierta a la fase de cierre, con un punto de apertura máxima que fue menos agudo (ver Figura 3 y 4).

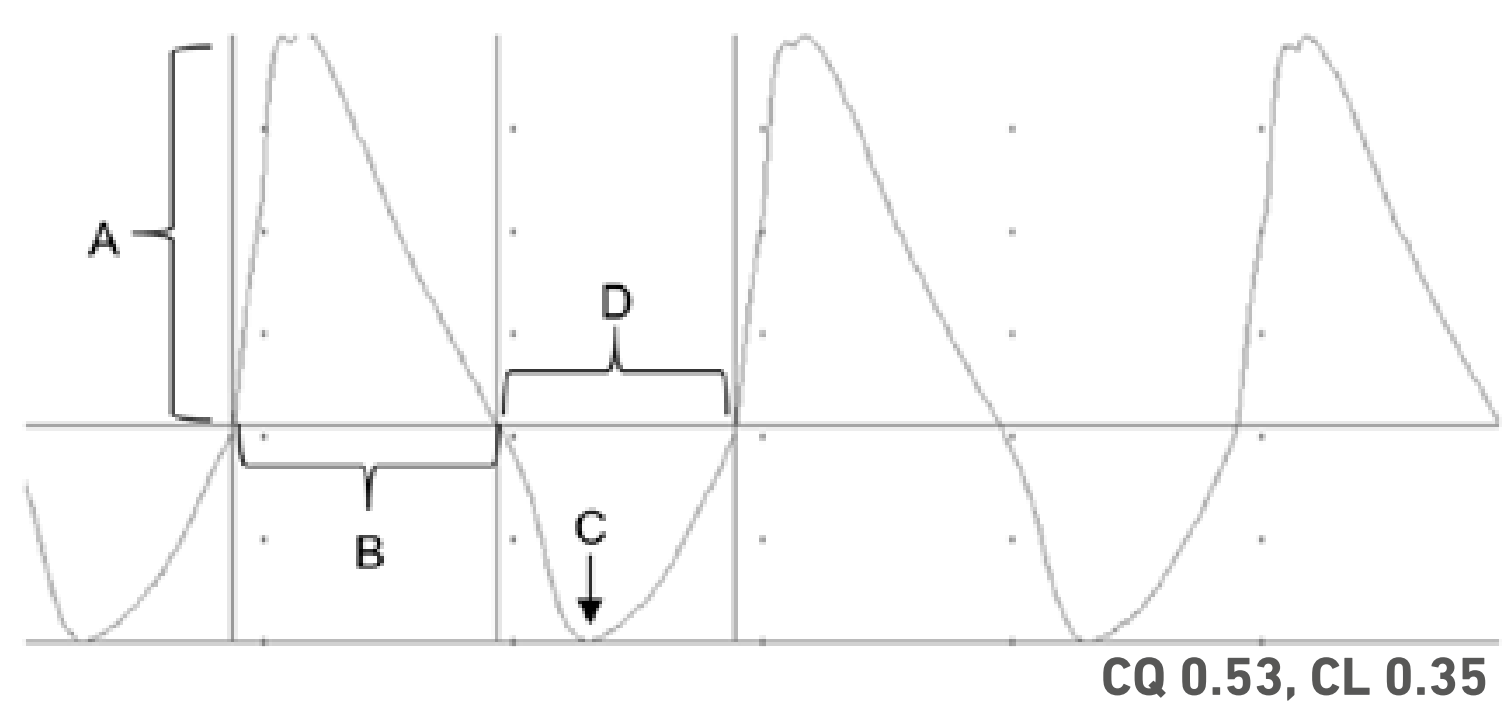

Figura 3. Onda EGG antes de la fonación en tubos

Nota: $A=$ zona de contacto glótico con mayor latencia. $B=$ perfil onda menos robusto en fase de cierre. $C=$ punto de apertura máxima más agudo. $D=$ Fase de apertura menos pronunciada . 


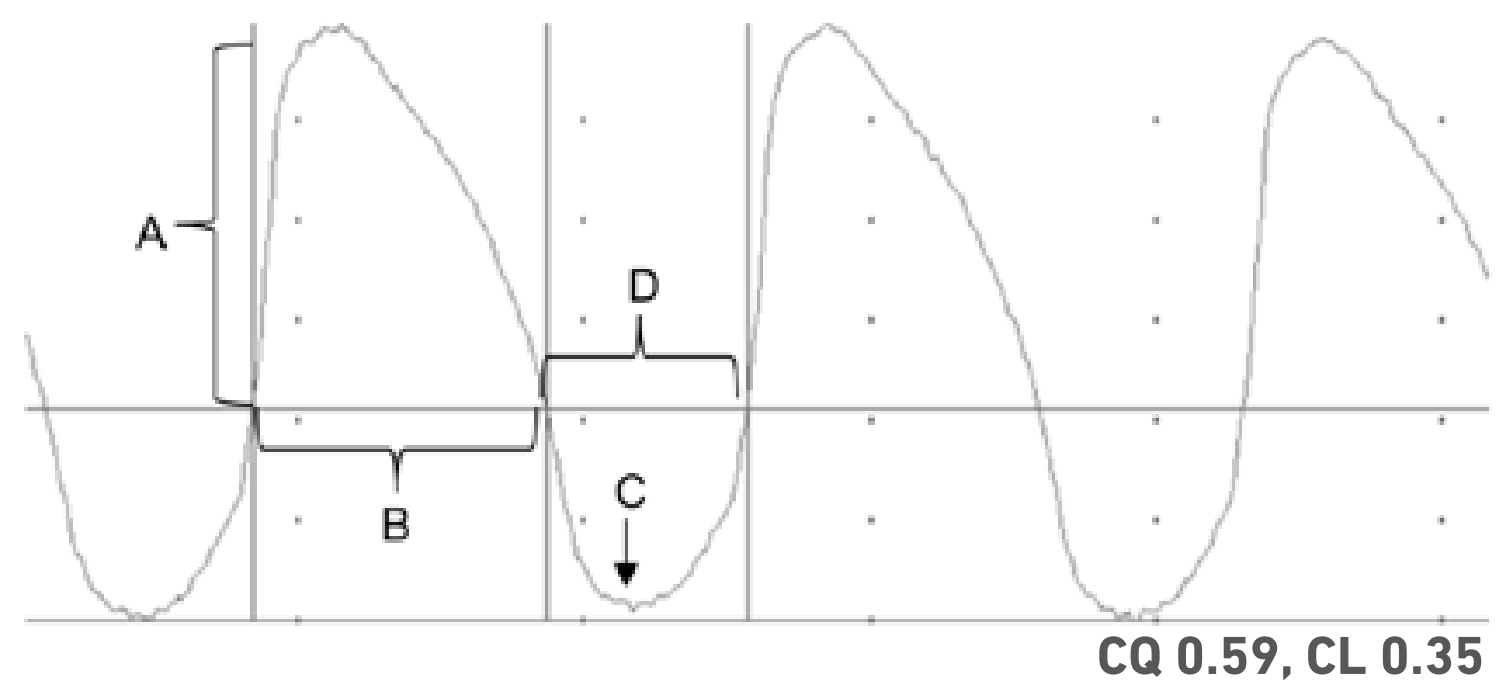

Figura 4. Onda EGG durante la fonación en tubos

Nota: $A=$ zona de contacto glótico con menor latencia. $B=$ perfil onda más robusto en fase de cierre. $C$ = punto de apertura máxima menos agudo. $D=$ Fase de apertura más pronunciada.

En cuanto al análisis de las ondas electroglotográficas durante la vibración lingual, puede afirmarse que antes de realizar el ejercicio, la amplitud de onda pico a pico se mantuvo en el mismo nivel a lo largo de los ciclos glóticos (Figura 5). Por el contrario, mientras los sujetos ejecutaban la actividad vocal, se observó una modificación oscilatoria de la amplitud pico. Dado que las muestras registradas eran de hombres y los periodos de onda eran tan extensos, fue necesario ampliar la ventana de análisis de $30 \mathrm{~ms}$ a $50 \mathrm{~ms}$ para poder observar el patrón. Este comportamiento fue homogéneo en la totalidad de las muestras evaluadas (ver Figura 6). La fase de cierre, el CQy la fase de apertura posterior al ejercicio permanecieron prácticamente inalterados en comparación con la fonación antes de la ejecución del ejercicio.

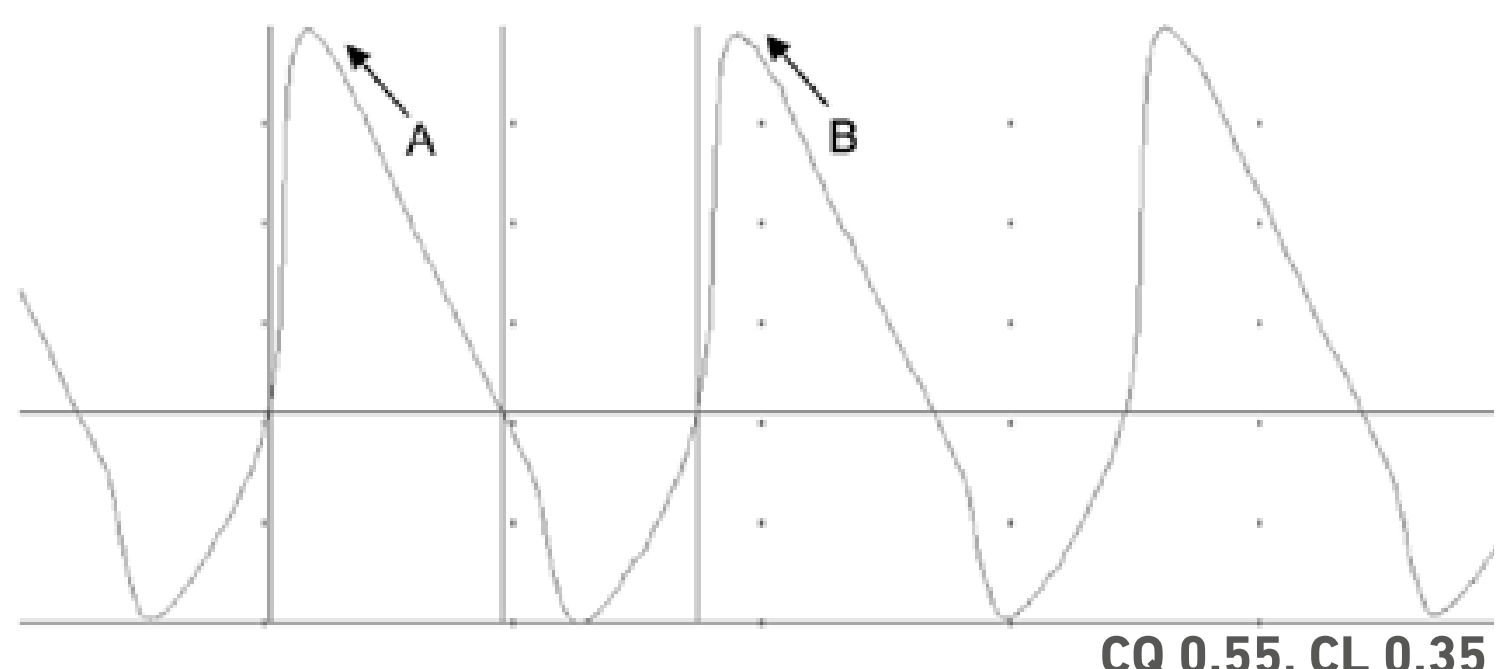

Figura 5. Onda EGG antes de la vibración lingual

Nota: $A$ y $B=$ estabilidad en la amplitud pico a pico entre una onda y otra. 


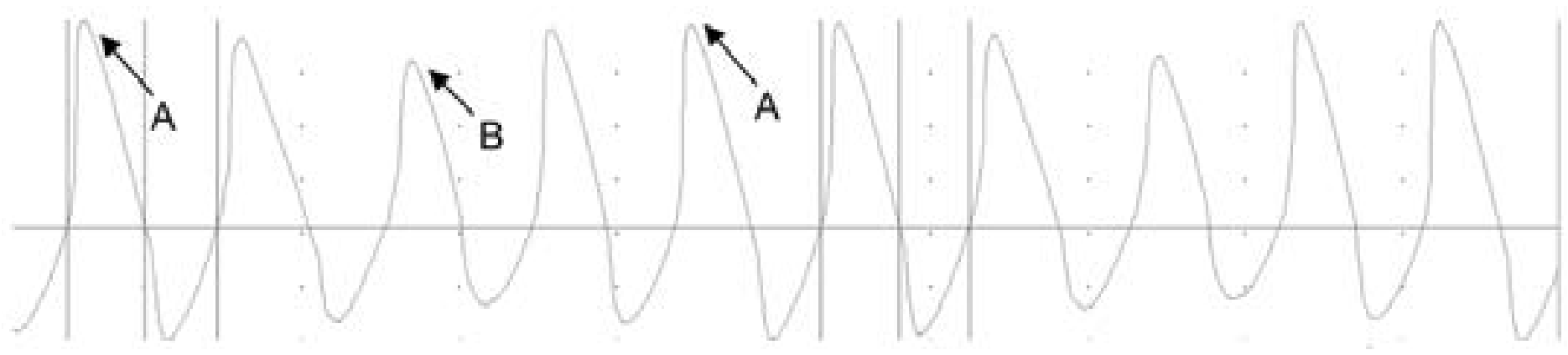

CQ 0.49, CL 0.35

Figura 6. Onda EGG durante la vibración lingual

Nota: $A=$ mayor amplitud pico a pico. $B=$ Menor amplitud pico a pico.

\section{Discusión}

\section{Comportamiento del CQ antes, durante y después de los ejercicios}

Este estudio examinó el efecto fisiológico de dos ejercicios con TVSO en la actividad laríngea de profesores con esfuerzo vocal constante. Los valores obtenidos del CQ antes, durante y después de la fonación en tubos indican diferencias significativas del parámetro durante la realización del ejercicio. Hasta la fecha, diversos estudios han explorado el efecto de la fonación en tubos en el CQ electroglotográfico, registrando este parámetro de forma aislada $[27,28,42,43]$ y junto a otros ejercicios con TVSO $[31,44,45]$. Para comenzar, en el estudio de Laukkanen et al. [42] se evidenció un aumento de la altura laríngea y consecuentemente una tendencia a aumentar el contacto glótico medido con EGG. Sin embargo, dado que la investigadora evaluó esta variable conjuntamente con electromiografía, sus conclusiones indican que el aumento del contacto glótico no necesariamente estuvo acompañado de un incremento de la contracción muscular y, por tanto, no indica una conducta hiperfuncional. Ya que los resultados de esta investigación muestran un aumento del CQ durante la realización del ejercicio, podría hipotetizarse que el incremento del parámetro no indica necesariamente la instauración de un patrón hiperfuncional. Sin embargo, es necesario confirmar este supuesto, dado que en este diseño experimental no se incluyeron medidas electromiográficas.

Igualmente, los resultados de este estudio concuerdan con los hallazgos de Gaskill y Quinney [28], debido a que el CQ aumentó durante el ejercicio y este mismo parámetro retornó a la línea base inmediatamente después de la realización de la tarea. Los autores hipotetizan que estos resultados se pueden deber a diversas causas. En primer lugar, el grado de resistencia generado por un tubo de diámetro pequeño puede provocar un aumento de la presión subglótica, con lo cual es necesario un aumento de la resistencia laríngea para mantener la vibración. Esto se traduce en mayor contacto de los pliegues vocales y por ende un aumento del CQ. Adicionalmente, en línea con los hallazgos del presente estudio, se observó una alta variabilidad de los datos; los autores insisten en las diferencias individuales, pues es posible que solamente algunos de los sujetos participantes, en su intento por sincronizar la fuente glótica con la impedancia generada por el tubo, evidencien efectos medibles a causa de la ejecución del ejercicio. Por otra parte, es necesario traer los análisis de Gaskill y Erickson [27] a esta 
discusión. Según proponen estos autores, para sincronizar la fuente glótica con la impedancia del tracto vocal, es necesario centrarse en las sensaciones percibidas durante la ejecución del ejercicio. Así pues, durante la actividad es posible que ocurran ajustes (o desajustes) individuales en la aducción laríngea o en la altura laríngea que contribuyen a modificaciones impredecibles en el CQ, las cuales pueden deberse a la reacción de los participantes frente a la presión intraroral generada por la resistencia al flujo. Esto es de particular importancia, puesto que se usaron los tubos con el diámetro más pequeño disponible comercialmente y por tanto son los tubos que generan mayor presión intraoral debido a la magnitud de la resistencia al flujo. Estudios que concuerdan con las conclusiones reportadas por los investigadores mencionados y que coinciden con los resultados de la presente investigación son los de Andrade et al. [15], Guzmán et al. [31,44] y Radolf et al. [45] quienes igualmente encontraron que la fonación en tubos tiende a aumentar el CQy que uno de los tubos que más generaba este efecto era el tubo de diámetro pequeño.

Con respecto a las modificaciones del CQ durante la vibración lingual, los resultados del presente estudio evidencian que el parámetro evaluado disminuye mientras se ejecuta el ejercicio; al terminar, el valor regresa a la línea base. Los estudios de Gaskill y Erickson [29] y de Hamdan et al. [30] analizaron estos ejercicios de forma aislada, mientras que Guzmán et al. [31,46] y Andrade et al. [15] observaron este ejercicio en conjunto con otras posturas de TVSO. Quienes observaron el ejercicio de forma aislada reportan entre sus hallazgos que el CQ decrece durante la realización del ejercicio y que este resultado es más evidente en sujetos con poco entrenamiento vocal. Esto pudo haber ocurrido en el presente estudio, puesto que los participantes no debían contar con entrenamiento vocal previo. Según los autores, esto puede deberse a un aumento del flujo de aire para mantener en vibración los dos cuerpos osciladores. Para ello existen dos posibles mecanismos: el primero, aumentando la presión subglótica; el segundo, disminuyendo la resistencia glótica. Asumiendo que la presión es constante, el CQ decrece por una disminución de la resistencia glótica, de modo que la única forma de realizar esta acción es abduciendo ligeramente los pliegues vocales y, por consiguiente, reduciendo el CQ [29]. Igualmente, durante el ejercicio ocurren aumentos intermitentes de la presión intraoral, que separan bruscamente los pliegues vocales durante la realización del ejercicio. Si se ejecuta con una fonación apretada, la vibración de la lengua va a ser difícil de mantener o incluso puede desaparecer [30].

Para el caso de ambos ejercicios, el hecho de que no se observen cambios posteriores a su realización puede deberse al corto tiempo de ejecución de la tarea. Es posible que 5 minutos no sean suficientes para instaurar y mantener un patrón fonatorio distinto. Por un lado, Gaskill \& Quinney [47] afirmaron que la relativa brevedad en la ejecución de los ejercicios podía interferir en la consecución de modificaciones en el patrón de aducción de los pliegues vocales que se mantenga luego de la ejecución de los ejercicios. Según las mismas autoras, este tiempo no es suficiente para sincronizar el comportamiento vocal con la resistencia del tubo y enfocarse en las sensaciones de resonancia anterior. Muchos sujetos requieren de más tiempo para encontrar la facilidad en la fonación y hallar sensaciones de resonancia. Puede ser que más tiempo de ejecución mostrara mejores resultados. Por otro lado, de acuerdo con los postulados del aprendizaje motor y la fisiología del ejercicio, se requieren múltiples repeticiones de la tarea motora para llegar incluso a la etapa más temprana de dominio motor, que es la de adquisición $[48,49]$. Los anteriores postulados deben estudiarse en el futuro. 


\section{Efecto del periodo y el tratamiento sobre el CQ}

Según los resultados del análisis de varianza (ANOVA), no existe un efecto del tratamiento sobre el comportamiento del CQ electroglotográfico. La interpretación de estos resultados debe realizarse con cautela, puesto que las pruebas de diferencia de medias indicaron que existe una tendencia clara de modificación del parámetro en cada uno de los ejercicios estudiados. La razón por la cual no existe un valor significativo que indique un efecto del tratamiento del CQ es porque debido a la alta variabilidad observada en los valores del CQ durante la ejecución de los ejercicios, los extremos de ambas poblaciones se solapan e impiden diferenciar el efecto de un tratamiento sobre el otro. El modelo ajustado indicó también que ninguno de los ejercicios presenta efectos residuales sobre el otro. Este resultado era esperable, pues al establecer la metodología de la presente investigación se determinó un tiempo de reposo vocal de quince minutos entre un ejercicio y otro. Este hallazgo confirma que un tiempo de descanso como el establecido en esta investigación es suficiente para eliminar los efectos logrados por un ejercicio cuando este es ejecutado por un tiempo máximo de 5 minutos.

\section{Modificaciones en el perfil de onda}

En términos generales, durante la fonación en tubos se observa que la consecución del contacto glótico máximo es más rápida en comparación con la emisión de la línea base. Esto puede indicar una mejoría en la oscilación autosostenida, principalmente del comportamiento de cierre glótico desde la porción inferior del pliegue vocal hasta la porción superior. De acuerdo con las interpretaciones de Titze [50], esto solo puede conseguirse cuando se modifican las presiones de aire en el tracto vocal y este es uno de los mecanismos de los ejercicios con TVSO. El principal hallazgo en el comportamiento de los perfiles de onda de EGG durante la realización de la vibración lingual fue la variación de la amplitud pico a pico a una frecuencia menor que la de emisión de los pliegues vocales (Figura 6). Este hallazgo ha sido reportado por varios autores entre los que pueden mencionarse Andrade et al. [15], Guzmán, [46] y Radolf et al. [45]. Los autores indican que la inducción de fluctuaciones en los registros de EGG es característico de los ejercicios con dos fuentes de vibración, debido a los aumentos momentáneos de la presión intraglótica, lo cual genera la oscilación de la magnitud de la amplitud pico a pico. Es posible que durante el aumento momentáneo de presión a lo largo de la oscilación del segundo cuerpo vibrante, se genere una separación de los pliegues vocales, que es registrada como una disminución de la impedancia. Adicionalmente, según indica Andrade et al. [15], estas oscilaciones son las que se perciben como efecto masaje durante la realización de ejercicios con dos fuentes de vibración.

\section{Conclusiones}

Los ejercicios con TVSO evaluados en el presente estudio demuestran un efecto diferencial en el CQ durante su realización. No obstante, el efecto es diferente dependiendo de si el ejercicio es de una fuente o de dos fuentes de vibración. Por un lado, el ejercicio de una fuente de vibración aumenta el CQ, lo que posiblemente se debe al aumento considerable de la resistencia al flujo. En contraposición, el ejercicio de dos fuentes de vibración disminuye el CQ. Esto puede deberse a un aumento del flujo de aire para mantener en vibración los dos cuerpos osciladores o a los cambios de presión que separan bruscamente los pliegues vocales durante la realización del ejercicio. Al parecer, cuando el ejercicio se ejecuta en tiempos tan cortos, los cambios conseguidos durante su realización no se mantienen en las emisiones de voz posteriores. 
Es igualmente necesario mencionar algunas limitaciones. Para empezar, aunque el estatus de saludables fue comprobado mediante el Índice de Discapacidad Vocal y las evaluaciones audioperceptuales, se reconoce que la forma idónea para el establecimiento del estatus laríngeo previo a la participación del experimento es mediante la realización de estudios imagenológicos de la laringe. En segundo lugar, el software utilizado para el análisis de los datos de EGG demostró una limitación para establecer el nivel de criterio estándar especialmente en zonas de la onda donde la amplitud pico a pico variaba. Esto fue particularmente evidente en los análisis de las ondas de EGG recolectadas durante la ejecución de los ejercicios con dos fuentes de vibración. Para solventar este inconveniente se excluyeron del análisis aquellos fragmentos de la onda donde la amplitud pico a pico disminuyó. Esto último para cumplir plenamente con los requisitos de análisis establecidos por el software. Es necesario contemplar en futuros estudios equipos de medición que permitan un análisis individualizado de cada ciclo, para tener en cuenta aquellas zonas de la onda con variaciones importantes que pueden impactar los resultados derivados de su análisis. Para terminar, en el presente estudio no se tuvo en cuenta la actividad vocal previa a la realización del experimento, ni el consumo de medicamentos por parte de los participantes; estas son posibles causas de la alta variabilidad observada en las mediciones obtenidas de ambos ejercicios, por lo que se sugiere contemplar y controlar estas variables intervinientes en estudios a futuro.

\section{Referencias}

1. Vintturi J, Alku P, Lauri E-R, Sala E, Sihvo M, Vilkman E. Objective Analysis of Vocal Warm-Up with Special Reference to Ergonomic Factors. J Voice [Internet]. 2001;15:3653. doi: https://doi.org/10.1016/S0892-1997(01)00005-4

2. Vilkman E. Voice problems at work: A challenge for occupational safety and health arrangement. Folia Phoniatr Logop [nternet]. 2000;52:120-5. doi: https://doi. org/10.1159/000021519

3. Cantor Cutiva LC, Vogel I, Burdorf A. Voice disorders in teachers and their associations with work-related factors: A systematic review. J. Commun. Disord [Internet]. 2013;14355. doi: https://doi.org/10.1016/j.jcomdis.2013.01.001

4. Munier C, Farrell R. Working Conditions and Workplace Barriers to Vocal Health in Primary School Teachers. J Voice [Internet]. 2016;30:127.e31-127.e41. doi: http://dx.doi. org/10.1016/j.jvoice.2015.03.004

5. Martins RHG, Pereira ERBN, Hidalgo CB, Tavares ELM. Voice Disorders in Teachers. A Review. J Voice [Internet]. 2014;28:716-24. doi: https://doi.org/10.1016/j.jvoice.2014.02.008

6. Cantor Cutiva LC, Burdorf A. Medical Costs and Productivity Costs Related to Voice Symptoms in Colombian Teachers. J Voice [Internet]. 2015;29:776.e15-776.e22. doi: http://dx.doi.org/10.1016/j.jvoice.2015.01.005

7. Atará-Paraquive ÁP, Ángel-Gordillo LF. Amplificación vocal en el ámbito ocupacional educativo: una revisión de la literatura. Rev la Fac Med [Internet]. 2018;66:485-93. Disponible en: https://revistas.unal.edu.co/index.php/revfacmed/article/view/58517

8. Titze IR. Voice Training and Therapy With a Semi-Occluded Vocal Tract: Rationale and Scientific Underpinnings. J Speech, Lang Hear Res [Internet]. 2006;49:448-59. doi: https://doi.org/10.1044/1092-4388(2006/035) 
9. Story BH, Laukkanen A-M, Titze IR. Acoustic impedance of an artificially lengthened and constricted vocal tract. J Voice [Internet]. 2000;14:455-69. doi: https://doi. org/10.1016/s0892-1997(00)80003-x

10. Titze IR. Phonation into a straw as a voice building exercise. J Sing. 2000;57:27-8.

11. Guzman M, Rubin A, Muñoz D, Jackson-Menaldi C. Changes in Glottal Contact Quotient During Resonance Tube Phonation and Phonation With Vibrato. J Voice [Internet]. 2013;27:305-11. doi: https://doi.org/10.1016/j.jvoice.2013.01.017

12. Titze IR, Laukkanen A-M. Can vocal economy in phonation be increased with an artificially lengthened vocal tract? A computer modeling study. Logop Phoniatr Vocology [Internet]. 2007;32:147-56. doi: https://doi.org/10.1080/14015430701439765

13. Simberg S, Sala E, Tuomainen J, Sellman J, Rönnemaa A-M. The Effectiveness of Group Therapy for Students With Mild Voice Disorders: A Controlled Clinical Trial. J Voice [Internet]. 2006;20:97-109. doi: https://doi.org/10.1016/j.jvoice.2005.01.002

14. Amarante Andrade P, Wistbacka G, Larsson H, Södersten M, Hammarberg B, Simberg $\mathrm{S}$, et al. The Flow and Pressure Relationships in Different Tubes Commonly Used for Semi-occluded Vocal Tract Exercises. J Voice [Internet]. 2016;30:36-41. doi: https://doi. org/10.1016/j.jvoice.2015.02.004

15. Andrade PA, Wood G, Ratcliffe P, Epstein R, Pijper A, Svec JG. Electroglottographic Study of Seven Semi-Occluded Exercises: LaxVox, Straw, Lip-Trill, Tongue-Trill, Humming, Hand-Over-Mouth, and Tongue-Trill Combined With Hand-Over-Mouth.J Voice [nternet]. 2014;28:589-95. doi: https://doi.org/10.1016/j.jvoice.2013.11.004

16. Maxfield L, Titze I, Hunter E, Kapsner-Smith M. Intraoral pressures produced by thirteen semi-occluded vocal tract gestures. Logop Phoniatr Vocology [Internet]. 2015;40:8692. doi: https://doi.org/10.3109/14015439.2014.913074

17. Vlot C, Ogawa M, Hosokawa K, Iwahashi T, Kato C, Inohara H. Investigation of the immediate effects of humming on vocal fold vibration irregularity using electroglottography and high-speed laryngoscopy in patients with organic voice disorders. J Voice [Internet]. 2017;31:48-56. doi: http://dx.doi.org/10.1016/j.jvoice.2016.03.010

18. Ogawa M, Hosokawa K, Yoshida M, Iwahashi T, Hashimoto M, Inohara H. Immediate Effects of Humming on Computed Electroglottographic Parameters in Patients With Muscle Tension Dysphonia. J Voice [Internet]. 2014;28:733-41. doi: http://dx.doi.org/10.1016/j.jvoice.2014.02.004

19. Laukkanen A-M, Titze IR, Hoffman H, Finnegan E. Effects of a Semioccluded Vocal Tract on Laryngeal Muscle Activity and Glottal Adduction in a Single Female Subject. Folia Phoniatr Logop [Internet]. 2008;60:298-31 1. doi: https://doi.org/10.1159/000170080

20. Yiu EM-L, Lo MCM, Barrett EA. A systematic review of resonant voice therapy. Int J Speech Lang Pathol [Internet]. 2017;19:17-29. doi: https://doi.org/10.1080/17549507. 2016.1226953

21. Cielo CA, Frigo LF, Christmann MK. Pressão sonora e tempo máximo de fonação após a técnica de finger kazoo. Rev CEFAC [Internet]. 2013;15:994-1000. doi: https://doi. org/10.1590/S1516-18462013000400029 
22. Cordeiro GF, Montagnoli AN, Ubrig MT, Menezes MHM, Tsuji DH. Comparison of Tongue and Lip Trills with Phonation of the Sustained Vowel / $\varepsilon /$ Regarding the Periodicity of the Electroglottographic Waveform and the Amplitude of the Electroglottographic Signal. Open J Acoust [Internet]. 2015;05:226-38. doi: http://dx.doi.org/10.4236/ oja.2015.54018

23. Cordeiro GF, Montagnoli AN, Nemr NK, Menezes MHM, Tsuji DH. Comparative Analysis of the Closed Quotient for Lip and Tongue Trills in Relation to the Sustained Vowel /ع/. J Voice [Internet]. 2012;26:e17-22. doi: https://doi.org/10.1016/j.jvoice.2010.07.004

24. Denizoglu II, Sahin M, Bayrak S, Uygun MN. Efficacy of Doctorvox Voice Therapy Technique for Mutational Falsetto. J Voice [Internet]. 2019;33:950.e1-950.e8. doi: https:// doi.org/10.1016/j.jvoice.2018.05.012

25. Saters TL, Ribeiro VV, Thaís L, Siqueira D, Marotti BD, Brasolotto AG, et al. The Voiced Oral High-frequency Oscillation Technique 's Immediate Effect on Individuals With Dysphonic and Normal Voices. J Voice [nternet]. 2017;32:449-58. doi: https://doi.org/10.1016/j.jvoice.2017.06.018

26. Laukkanen A-M. About the so called "resonance tubes" used in Finnish voice training practice: An electroglottographic and acoustic investigation on the effects of this method on the voice quality of subjects with normal voice. Scand J Logop Phoniatr [Internet]. 1992;17:151-61. doi: https://doi.org/10.3109/14015439209098733

27. Gaskill CS, Erickson ML. The effect of an artificially lengthened vocal tract on estimated glottal contact quotient in untrained male voices. J Voice [Internet]. 2010;24:57-71. doi: http://dx.doi.org/10.1016/j.jvoice.2008.05.004

28. Gaskill CS, Quinney DM. The effect of resonance tubes on glottal contact quotient with and without task instruction: A comparison of trained and untrained voices. J Voice [Internet]. 2012;26:e79-93. doi: http://dx.doi.org/10.1016/j.jvoice.2011.03.003

29. Gaskill CS, Erickson ML. The Effect of a Voiced Lip Trill on Estimated Glottal Closed Quotient. J Voice [Internet]. 2008;22:634-43. doi: http://dx.doi.org/10.1016/j.jvoice.2007.03.012

30. Hamdan AL, Nassar J, Al Zaghal Z, El-Khoury E, Bsat M, Tabri D. Glottal contact quotient in Mediterranean tongue trill. J Voice [Internet]. 2012;26:669.e1 1-669.e15. doi: http://dx.doi.org/10.1016/j.jvoice.2011.07.008

31. Guzman M, Calvache C, Romero L, Muñoz D, Olavarria C, Madrid S, et al. Do Different Semi-Occluded Voice Exercises Affect Vocal Fold Adduction Differently in Subjects Diagnosed with Hyperfunctional Dysphonia? Folia Phoniatr Logop [Internet]. 2015;67:68-75. doi: https://doi.org/10.1159/000437353

32. Mailänder E, Mühre L, Barsties B. LaxVox as a voice training program for teachers: a pilot study. J voice [Internet]. 2017;31:262.e13-262.e22. doi: https://doi.org/10.1016/j. jvoice.2016.04.011

33. Calvache-Mora CA CA. Efectividad del calentamiento vocal fisiológico para cantantes. Ciencias la Salud [Internet]. 2016;14:367-79. Disponible en: http://revistas.urosario. edu.co/index.php/revsalud/article/view/5145/3505 
34. Calvache G, Guzman M, Bobadilla M, Bortnem G. Variation on Vocal Economy After Different Semioccluded Vocal Tract Exercises in Subjects With Normal Voice and Dysphonia. J Voice [Internet]. 2020;34:582-9. doi: https://doi.org/10.1016/j.jvoice.2019.01.007

35. Zamponi V, Mazzilli R, Mazzilli F, Fantini M. Effect of sex hormones on human voice physiology: from childhood to senescence. Hormones [Internet]. 2021;20:691-6. Disponible en: https://link.springer.com/10.1007/s42000-021-00298-y

36. Nanjundeswaran C, Li NYK, Chan KMK, Wong RKS, Yiu EM-L, Verdolini-Abbott K. Preliminary Data on Prevention and Treatment of Voice Problems in Student Teachers. J Voice [Internet]. 2012;26:816.e1-816.e12. Disponible en: https://linkinghub.elsevier. com/retrieve/pii/S0892199712000665

37. Jacobson BH, Johnson A, Grywalski C, Silbergleit A, Jacobson G, Benninger MS, et al. The Voice Handicap Index (VHI). Am J Speech-Language Pathol [Internet]. 1997;6:6670. doi: https://doi.org/10.1044/1058-0360.0603.66

38. Núñez-Batalla F, Corte-Santos P, Señaris-González B, Llorente-Pendás JL, Górriz-Gil C, Suárez-Nieto C. Adaptación y validación del índice de incapacidad vocal (VHI-30) y su versión abreviada (VHI-10) al español. Acta Otorrinolaringológica Española [Internet]. 2007;58:386-92. Disponible en: https://linkinghub.elsevier.com/retrieve/pii/ S0001651907749543

39. Howard DM. Electroglottography/electrolaryngography. In: Fried M, Ferlito A, editores. The larynx. San Diego: Plural Press; 2008. p. 1-17.

40. Miller D. Voce Vista [nternet]. 2017. Disponible en: http://www.vocevista.com/

41. Rothenberg M, MahshieJJ. Monitoring Vocal Fold Abduction through Vocal Fold Contact Area. J Speech Lang Hear Res [nternet]. 1988;31:338. doi: https://doi.org/10.1044/ jshr.3103.338

42. Laukkanen A-M, Lindholm P, Vilkman E. Phonation into a Tube as a Voice Training Method: Acoustic and Physiologic Observations. Folia Phoniatr Logop [Internet]. 1995;47:331-8. doi: https://doi.org/10.1159/000266371

43. Mills RD, Rivedal S, DeMorett C, Maples G, Jiang JJ. Effects of straw phonation through tubes of varied lengths on sustained vowels in normal-voiced participants. J Voice [Internet]. 2017;32(3):386. doi: http://dx.doi.org/10.1016/j.jvoice.2017.05.015

44. Guzmán M, Castro C, Madrid S, Olavarria C, Leiva M, Muñoz D, et al. Air Pressure and Contact Quotient Measures During Different Semioccluded Postures in Subjects With Different Voice Conditions. J Voice [Internet]. 2016;30:759.e1-759.e10. doi: http://dx. doi.org/10.1016/j.jvoice.2015.09.010

45. Radolf V, Laukkanen AM, Horáček J, Liu D. Air-pressure, vocal fold vibration and acoustic characteristics of phonation during vocal exercising $\cdot$ part $1:$ measurement in vivo. Eng Mech [Internet]. 2014;21:53-9. doi: https://doi.org/10.1016/j.jvoice.2016.10.024 
46. Guzman M, Jara R, Olavarria C, Caceres P, Escuti G, Medina F, et al. Efficacy of Water Resistance Therapy in Subjects Diagnosed With Behavioral Dysphonia: A Randomized Controlled Trial. J Voice [nternet]. Elsevier Inc.; 2017;31:385.e1-385.e10. doi: http:// dx.doi.org/10.1016/j.jvoice.2016.09.005

47. Gaskill CS, O'Brien SG, Tinter SR. The Effect of Voice Amplification on Occupational Vocal Dose in Elementary School Teachers. J Voice [Internet]. 2012;26:667.e19-667.e27. Disponible en: https://linkinghub.elsevier.com/retrieve/pii/S0892199711002001

48. Stathopoulos E, Felson Duchan J. History and Principles of Exercise-Based Therapy: How They Inform Our Current Treatment. Semin Speech Lang [Internet]. 2006;27:22735. Disponible en: http://www.thieme-connect.de/DOI/DOI? 10.1055/s-2006-955113

49. Delprado-Aguirre F. Fisiología del ejercicio en la práctica de la vocología colombiana: una necesidad. Rev Investig e Innovación en Ciencias la Salud [Internet]. 2020;2:44-55. Disponible en: http://revistas.fumc.edu.co:8080/ojs/index.php/RCMC/article/view/31

50. Titze IR. Principles of voice production. 2 ed. Iowa: Prentice-Hall; 2000. 